\title{
COMPOSICIÓN QUÍMICA DE LA MORERA (Morus alba), PARA USO EN LA ALIMENTACIÓN ANIMAL: DENSIDADES Y FRECUENCIAS DE PODA 1
}

\author{
Carlos Boschini F. ${ }^{2}$, Herbert Dormond H. ${ }^{2}$, Alvaro CastroH. ${ }^{3}$
}

\begin{abstract}
RESUMEN
Composición química de la morera (Morus alba), para uso en la alimentación animal: densidades y frecuencias de poda. El presente trabajo se llevó a cabo en la Estación Experimental de Ganado Lechero "Alfredo Volio Mata", Cartago, Costa Rica. Las muestras fueron obtenidas de una plantación de morera dividida en tres lotes, con un distanciamiento entre plantas de 60, 90 y $120 \mathrm{~cm}$ entre hileras y plantas. Cada parcela se uniformó en dos alturas de poda, 30 y $60 \mathrm{~cm}$ sobre el nivel del suelo. Las subparcelas se podaron en forma consecutiva cada 56, 84 y 112 días durante 336 días. Los factores distancia se siembra, altura de poda y frecuencia de corte, influyeron sobre los contenidos químicos de la materia seca. La distancia de siembra y la altura de poda produjeron pequeñas variaciones en la composición de la hojas y en los tallos de la morera. La frecuencia de corte produjo cambios en la composición del las hojas y tallos de la morera; sin embargo, las diferencias en materia seca, proteína, carbohidratos estructurales o en las cenizas totales de las hojas no fueron superiores al $3 \%$ entre las frecuencias experimentadas. En el tallo los cambios mayores fueron de un $9 \%$ y se produjeron en los contenidos de materia seca, pared celular y en algunos de sus constituyentes estructurales como la celulosa. El número de corte dentro de cada frecuencia influyó de manera significativa sobre las proporciones químicas de hojas y tallos.
\end{abstract}

\begin{abstract}
Chemical composition of mulberry (Morus alba) for use in animal feed: densities and proing frequencies. This experiment was carried out at the Alfredo Volio Mata Dairy Cattle Experimental Station in Costa Rica, at an altitude of 1542 masl, where there is an average annual rainfall of $2050 \mathrm{~mm}$, temperature of $19.5 \mathrm{C}$ and relative humidity of $84 \%$. A mulberry bush plantation was divided into three plots: with a distance of 60,90 and $120 \mathrm{~cm}$ between rows and plants. Each plot was uniformly pruned at two heights: 30 and $60 \mathrm{~cm}$ from the ground. During a 336-day period, these subplots were pruned consecutively every 56th, 84th and 112th day. The planting distance and the pruning height produced small variations in the chemical composition of the mulberry leaves and stems. The cutting frequency produced changes in the composition of the leaves and stems. Nevertheless, the differences in dry matter, crude protein, structural carbohydrates and total ashes in the leaves were not higher then $3 \%$ in experimental frequencies. In the stems the greatest changes were $9 \%$ and they were produced in the content of the dry matter, cellular wall and in some of the structural chemical components, such as cellulose. The cutting number within each frequency significantly influenced the chemical composition of the leaves and the stems.
\end{abstract}

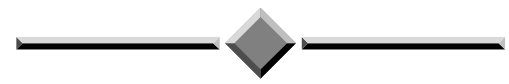

\section{INTRODUCCIÓN}

La ganadería de leche enfrenta el reto de hacer más eficaz los sistemas de producción y alimentación. Se espera que los modelos del futuro tengan como base algún enfoque de sostenibilidad y un uso racional de los recursos naturales (Castro y Benavides, 1994), especialmente aquellos con mayor aprovechamiento bio- lógico y económico. La morera, aunque originaria de Asia, se ha adaptado de manera excelente en la zona tropical de Centroamérica (Benavides et al., 1994; Rodríguez et al., 1994; Boschini, et al., 1998) con rendimientos muy altos. En cultivo para la producción de forraje, la morera ha mostrado excelentes características de palatabilidad y en consecuencia un alto consumo por el ganado (Benavides et al., 1994; Ortiz, 1992;

\footnotetext{
1 Inscrito en la Vicerrectoría de Investigación. No. 737-93-331.

2 Estación Experimental Alfredo Volio Mata, Facultad de Agronomía, Universidad de Costa Rica.

3 Programa Ganado Caprimo. Ministerio de Agricultura y Ganadería. Gobiero de Costa Rica.
} 
Castro, 1989). La literatura indica que la planta entera tiene un contenido protéico que varía entre 14 a $22 \%$ de proteína cruda en base seca (Piccioni, 1970) y la digestibilidad in vitro va de 70 a $80 \%$ (Ortiz, 1992). La muestras de morera analizadas por UFAG Laboratorien de Suiza, provenientes de la zona alta de Cartago en la Meseta Central de Costa Rica, presentaron valores en base seca de $22 \%$ de proteína cruda, $19 \%$ de fibra cruda, $2,3 \%$ de extracto etéreo, $50 \%$ de proteína sobrepasante y una estimación de $1,48 \mathrm{Mcal} / \mathrm{kg}$ de energía neta para lactación. Eswara y Reddy 1992, evaluando el valor nutritivo de la hojas de morera, han informadodo consumos de materia seca de 2,74 a $3,55 \%$ del peso vivo en cabras y ovejas, con contenidos de $12 \%$ de proteina cruda y $71 \%$ de TND.

El valor bromatológico de la morera radica especialmente en la hoja. Sin embargo, por ser una planta arbustiva, el suministro de la biomasa se torna en un aspecto importante para el mejor aprovechamiento en la alimentación animal. Boschini et al., 1998, han informadodo relaciones hoja : tallo superiores a 1 antes de los 100 días de crecimiento. Las proporciones varían de acuerdo a las condiciones de explotación y manejo, especialmente la densidad de plantas por área y la frecuencia de corte influyen en forma significativa. En el ganado bovino, se ofrece al animal la planta entera la morera, previamente picada. Esto constituye una mezcla de hoja y tallos que el animal selecciona durante el consumo. Por esta razón es necesario conocer en forma precisa la composición bromatológica de las hojas y tallos. El presente trabajo tuvo el propósito de buscar ese objetivo, tomando en cuenta tres distancias de siembra, dos alturas de corte y tres frecuencias de poda, realizadas a través de un ciclo de producción anual.

\section{MATERIALES Y MÉTODOS}

El experimento se llevó a cabo en la Estación Experimental de Ganado Lechero "Alfredo Volio Mata" de la Universidad de Costa Rica. La Estación está ubicada a $1542 \mathrm{msnm}$, con una precipitación anual media de $2050 \mathrm{~mm}$, distribuida durante los meses de mayo a noviembre. De diciembre a mediados de mayo la precipitación pluvial decae completamente. La temperatura media es de $19.5^{\circ} \mathrm{C}$ y la humedad relativa media es de $84 \%$. El suelo es de origen volcánico, clasificado como Typic Distrandepts (Vásquez, 1982). Se caracteriza por una profundidad media, con buen drenaje natural, una fertilidad media (7.7 de calcio, 3.0 de magnesio y 1.54 $\mathrm{cmol} / 1$ de potasio, 10.0 de fósforo, 28.8 de cobre, 234 de hierro, 6.3 de manganeso y $2.6 \mathrm{mg} / 1 \mathrm{de}$ zinc). Un $\mathrm{pH}$ de 5,9. Ecológicamente, la zona se tipifica como bosque húmedo montano bajo (Tosi, 1970, citado por Vásquez, 1982).
Las labores agrícolas y condiciones de siembra y manejo fueron descritas por Boschini et al., 1998. El cultivo se desarrolló durante un año, período en el cual se realizó el control de malas hierbas y se fertilizó con nitrato de amonio en julio y octubre, en porciones iguales, hasta completar la dosis de $150 \mathrm{~kg}$ de N/ha/año. En mayo del año siguiente, los lotes recibieron una poda de uniformización, realizada con tijera, una mitad de cada lote a 30 y la otra mitad a $60 \mathrm{~cm}$ de altura sobre el nivel del suelo. A partir de la fecha de uniformización, se programaron 6 cortes consecutivos cada 56, cuatro cada 84 y tres cada 112 días, durante 336 días experimentales. Después de cada corte, se controló la hierba existente, dejándo el material sobrante entre las hileras. Cuando el rebrote de la morera alcanzó tres-cinco $\mathrm{cm}$ de largo (aprox. dos semanas post corte), se fertilizó con nitrato de amonio, en la fracción correspondiente, a una dosis de $150 \mathrm{~kg} \mathrm{~N} / \mathrm{ha} / \mathrm{año}$ (Rodríguez et al, 1994). La dosis anual fue de $300 \mathrm{~kg} / \mathrm{ha}$.

Se empleó un modelo de parcelas divididas, con observaciones desigualmente repetidas en el tiempo. Las parcelas grandes correspondieron a las distancias entre plantas, las sub-parcelas a las alturas de corte y las sub-parcelas de esta a las frecuencias de corte, con la siguiente estructura estadística:

$\mathrm{Y}_{\mathrm{ijkl}}=\mathrm{U}+\mathrm{D}_{\mathrm{i}}+\mathrm{A}_{\mathrm{j}}+\mathrm{F}_{\mathrm{k}}+\mathrm{CF}_{\mathrm{l}(\mathrm{k})}+\mathrm{DA}_{\mathrm{ij}}+\mathrm{DF}_{\mathrm{jk}}+\mathrm{AF}_{\mathrm{ijk}}+$
$\mathrm{CDF}_{\mathrm{l}(\mathrm{ik})}+\mathrm{DAF}_{\mathrm{ijk}}+\mathrm{E}_{\mathrm{ijkl}}$. donde :

Yijkl = Porcentaje de materia seca y los contenidos químicos en la i-ésima distancia de siembra, j-ésima altura de poda, k-ésima frecuencia de corte y l-ésimo número de poda.

$\mathrm{U}=$ Media poblacional.

Di $\quad=$ Efecto de la distancia de siembra $(60,90$, $120 \mathrm{~cm}$ )

$\mathrm{Aj} \quad=$ Efecto de altura de poda $(30 \mathrm{y} 60 \mathrm{~cm})$.

$\mathrm{Fk} \quad=$ Efecto de la frecuencia de corte $(56,84$, 112 días).

$\mathrm{CFl}(\mathrm{k})=$ Efecto del número de poda dentro de cada frecuencia de corte.

DAij = Interacción de la distancia de siembra por altura de poda.

DFik = Interacción de la distancia de siembra por frecuencia de corte.

AFjk = Interacción de la altura de poda por frecuencia de corte.

$\mathrm{CDFl}(\mathrm{ik})=$ Efecto del número de cortes dentro de distancia de siembra por frecuencia de corte.

DAFijk = Interacción de la distancia de siembra por altura de poda por frecuencia de corte.

Eijkl. $=$ Error experimental. 
Las muestras de morera fueron recolectadas en cada distancia de siembra (parcela) y altura de corte, en la fecha de poda correspondiente a la frecuencia de corte programada. Las ramas cortadas fueron pesadas en fresco en el campo y se procedió a separar hojas y tallos para su cuantificación y análisis. Cada muestra fue secada en un horno a $60{ }^{\circ} \mathrm{C}$ durante 48 horas, hasta alcanzar un peso constante. Posteriormente se molieron y se procedió a determinar el contenido de materia seca en una estufa a $105{ }^{\mathrm{a}} \mathrm{C}$, la proteína cruda por el método Kjeldall y la cenizas totales (AOAC, 1980). La fibra neutro detergente (FND), la fibra ácido detergente (FAD), la lignina fueron analizadas por el método descrito por Goering y Van Soest (1970). Los valores de hemicelulosa y celulosa se obtuvieron por diferencia de las fracciones correspondientes usando el modelo estadístico descrito previamente. El contenido de materia seca y las fracciones químicas detereminadas se analizaron con el PROC GLM del paquete estadístico SAS (1985). Las fuentes de variación que resultaron estadísticamente, se sometieron a la prueba de Duncan para diferenciar la significancia entre medias.

\section{RESULTADOS}

La composición química de las hojas y tallos de morera para los efectos independientes: distancia de siembra, altura de poda y frecuencia de corte, se presenta en el Cuadro 1. En las hojas, el contenido de materia seca en base fresca fue muy constante $(\mathrm{P}>0,05)$ para las distancias de siembra experimentadas. La altura de poda y la frecuencia de corte influyeron significativamente $(\mathrm{P}<0,05)$; sin embargo la diferencia de materia seca entre 30 y $60 \mathrm{~cm}$ de altura de poda fue menor a $1 \%$ y entre las frecuencias de corte, no se diferencia entre 56 y 84 días y alrededor de $2.5-3 \%$ entre ellas y los 112 días. El contenido de proteína cruda mostró muy pequeñas diferencias $(\mathrm{P}<0,05)$ entre las distancias de siembra y entre las alturas de poda. Estas variaciones se acentuaron $(\mathrm{P}<0,01)$ entre las frecuencias de corte, disminuyendo $2-3 \%$ cada 28 días a partir de las 8 semanas de crecimiento. Tanto la pared celular y la fibra ácida como sus carbohidratos estructurales hemicelulosa, celulosa, lignina y las cenizas totales no fueron afectadas $(\mathrm{P}>0,05)$ por las distancias de siembra. La pared celular y las cenizas totales mostraron muy pequeñas diferencias $(P>0,05)$ entre las alturas de corta. Los contenidos de hemicelulosa, fibra ácida, celulosa y lignina muestran diferencias no significativas $(\mathrm{P}>$ $0,05)$ entre las alturas. La frecuencua de corte influyó sobre la pared celular y las cenizas totales $(\mathrm{P}<0,01)$, así como sobre la fibra ácida y la celulosa $(\mathrm{P}<0,05)$. Las variaciones en los contenidos de hemicelulosa y lignina no fueron significativas $(\mathrm{P}>0,05)$ al variar la frecuencia de corte.
La interacción de las variables distancia de siembra por frecuencia de corte resultó significativa $(\mathrm{P}<0,01)$ para la concentración de materia seca en la hoja, su contenido proteico y mineral. Las fracciones estructurales no se afectaron $(\mathrm{P}>0,05)$ por el efecto conjunto (Cuadro 2). En el Cuadro 3, se presenta la composición química de las hojas y tallos de morera sembrados a diferentes distancias y podadas a diferentes alturas. El contenido de materia seca, proteína cruda, pared celular, la hemicelulosa y las cenizas totales recibieron un efecto de interacción $(\mathrm{P}<0.05)$ por acción de estas variables. La fibra ácida y sus componentes no fueron influidas. Al observar el efecto asociado de la altura de poda y la frecuencia con que llevó a cabo (ver Cuadro 4), sobre la composición química de la hoja, dio resultados significativos sobre el contenido de proteína cru$\mathrm{da}$, la pared celular $(\mathrm{P}<0,01)$ y las cenizas totales $(\mathrm{P}<0,01)$. El contenido de materia seca, los componentes de la fibra ácida y la hemicelulosa no mostraron variaciones importantes $(\mathrm{P}>0,05)$. En el Cuadro 5, se presentan los valores de los componentes químicos de la hoja, desglosados por el número de corte anidado dentro de cada frecuencia. El análisis mostró que este tuvo un efecto altamente influyente $(\mathrm{P}<0,01)$ sobre todos los componentes químicos estudiados. La interacción de mayor rango, distancia por altura por frecuencia de corte, influyó el contenido de materia seca, proteína cruda y cenizas totales $(\mathrm{P}<0,01)$. Ninguno de los carbohidratos estructurales mostraron variaciones de importancia.

En los tallos, la materia seca en base fresca no mostró variaciones de importancia $(\mathrm{P}>0,05)$ entre las distancias de siembra. Entre las alturas de poda hubo una diferencia $(\mathrm{P}<0,05)$ menor al $1 \%$ y en las frecuencias de corte, se manifestaron variaciones de gran importancia $(\mathrm{P}<0,01)$ entre ellas. El contenido de proteína cruda fue muy constante $(\mathrm{P}>0,05)$ entre las distancias de siembra y entre las alturas de poda. La frecuencia de corte influyó $(\mathrm{P}<0,01)$ de manera evidente. La pared celular, la fibra ácida y sus componentes: celulosa y lignina, no mostraron variaciones $(\mathrm{P}>$ $0,05)$ de importancia conforme cambia la distancia de siembra. La distancia influye sobre la hemicelulosa y las cenizas totales $(\mathrm{P}<0,05)$ dentro de un margen muy estrecho. La altura de poda no influyó sobre ninguno de los carbohidratos estructurales del tallo, ni sobre el contenido de cenizas totales. La frecuencia de corte tiene un efecto marcado $(\mathrm{P}<0,01)$ sobre la pared celular y sus componentes, y de igual forma influye sobre los valores de las cenizas totales.

La interacción de las variables distancia de siembra por frecuencia de corte (Cuadro 2) resultó significativa $(\mathrm{P}<0,01)$ para la concentración de materia seca en el tallo. El contenido proteico, las cenizas totales y las 


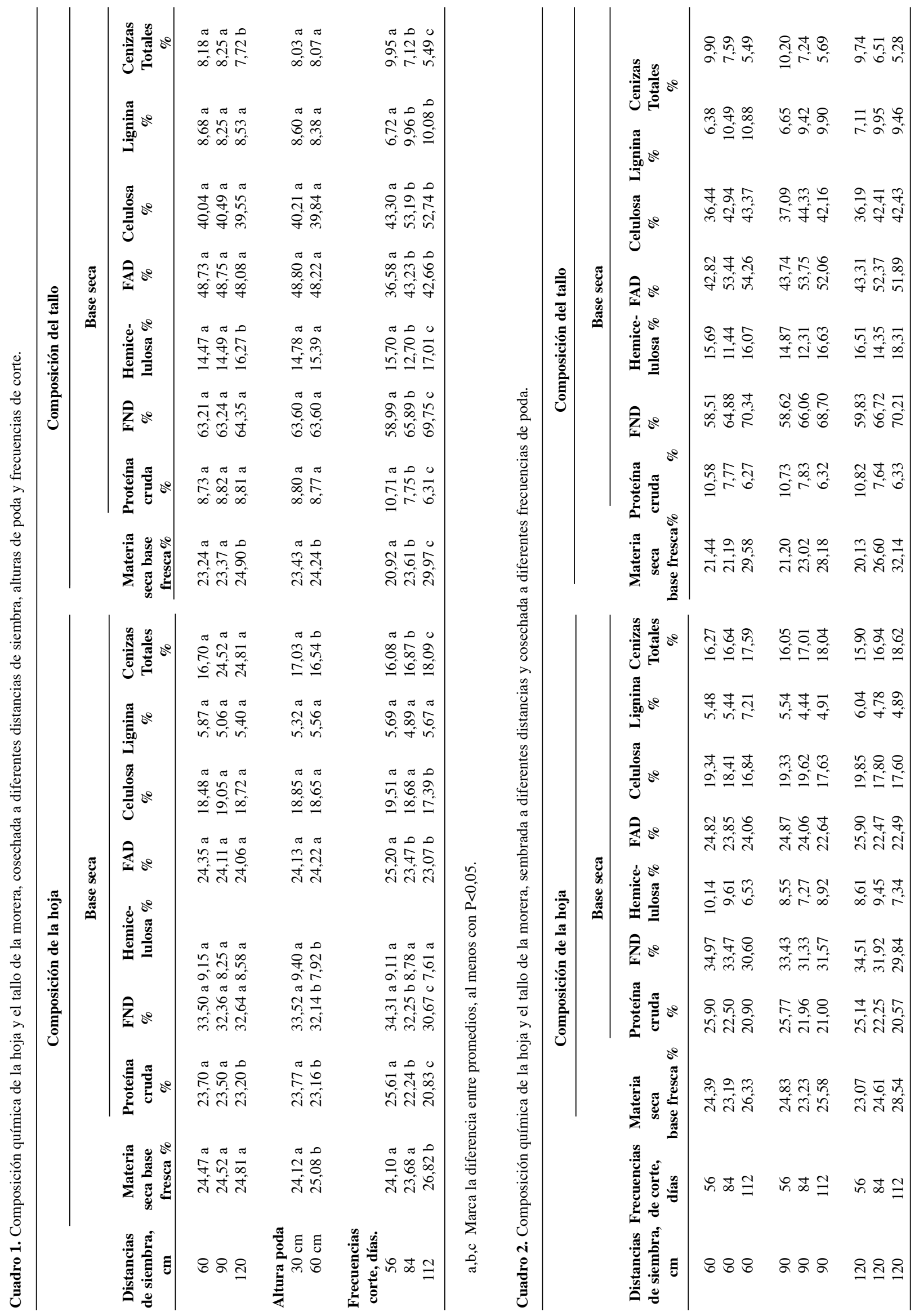


fracciones estructurales no parecen estar influidas $(\mathrm{P}>$ $0,05)$ por la acción conjunta de estos factores. En el Cuadro 3, se muestra la composición química del tallo en las plantas sembradas a diferentes distancias y podadas a diferentes alturas. Se observó que el contenido de hemicelulosa, fibra ácida y celulosa fueron influidos por la acción asociada de ambas variables en forma significativa $(\mathrm{P}<0,01)$. Los restantes componentes no recibieron ese efecto en forma evidente. Al analizar el efecto asociado de la altura de poda y la frecuencia de corte sobre la composición química del tallo, presentado en el Cuadro 4, se observó que únicamente el contenido de materia seca fue influido de manera evidente $(\mathrm{P}$ $<0,01)$. Ninguno de los componentes de la pared celular, ni el contenido de cenizas totales mostró variaciones de importancia $(\mathrm{P}>0,05)$ en el tallo. El Cuadro 5 presentan los valores de los componentes químicos del tallo, desagregando el número de corte anidado en cada intervalo de poda. El análisis mostró que el número de corte efectuado dentro de cada frecuencia tuvo un efecto altamente determinante $(\mathrm{P}<0,01)$ sobre todos los componentes químicos estudiados. La interacción entre los factores distancia de siembra por altura de poda y frecuencia de corte, influyó $(\mathrm{P}<0,05)$ solamente en el contenido de materia seca del tallo. La proteína cruda, la pared celular y sus componentes, así como el contenido de cenizas totales, no presentaron variaciones de importancia.

La composición química general (promedio y desviación estándar del error) en el experimento fue de $24,60 \%( \pm 2,24)$ de materia seca en la planta fresca, $23,46 \%( \pm 0,59)$ de proteína cruda, $32,83 \%( \pm 3,87)$ de fibra neutro detergente, $8,66 \%( \pm 3,85)$ de hemicelulosa, $24,17 \%( \pm 2,76)$ de fibra ácido detergente, $18,75 \%$ $( \pm 2,46)$ de celulosa, $5,44 \%( \pm 2,21)$ de lignina y $16,78 \%$ $( \pm 0,75)$ de cenizas totales en la hoja y de $23,83 \%$ $( \pm 2,01)$ de materia seca en la planta, $8,78 \%( \pm 0,66)$ de proteína cruda, $63,59 \%( \pm 2,76)$ de fibra neutro detergente, $15,07 \%( \pm 2,77)$ de hemicelulosa, $48,51 \%$ $( \pm 2,99)$ de fibra ácido detergente, $40,02 \%( \pm 2,78)$ de celulosa, $8,49 \%( \pm 1,13)$ de lignina y $8,04 \%( \pm 0,88)$ de cenizas en el tallo.

\section{DISCUSIÓN}

En el presente experimento se muestra la composición química de las hojas y tallos de la morera y las variaciones que produce los factores estudiados. La distancia de siembra entre plantas provocó variaciones estadísticamente significativas sobre el contenido de proteína cruda en la hoja y sobre la concentración de materia seca, hemicelulosa y cenizas en el tallo; sin embargo, las diferencias encontradas son pequeñas desde el punto de vista biológico. Los demás contituyentes analizados mostraron variaciones muy estrechas en relación al promedio general de cada componente. En términos generales, las distancias de siembra estudiadas no influyeron biológicamente sobre el contenido de nutrientes presentes de la hoja o el tallo de la morera. La altura de poda influyó estadísticamente también sobre la materia seca, la proteína, la pared celular, la fibra ácida y las cenizas totales en la hoja y únicamente sobre la materia seca en el tallo. A pesar de la manifestación de las variaciones estadísticas encontradas, las diferencias reales fueron ligeramente superiores al $1 \%$, lo que no constituye un efecto biológicamente importante para inclinarse favorablemente a una particular alturas de corte. Contrariamente a los resultados observados en las distancias de siembra y la altura de poda, la frecuencia de corte mostró un efecto de verdadera importancia sobre la acumulación de nutrientes, con excepción de hemicelulosa y lignina en la hoja. A bajos intervalos de poda la concentración de proteína es alta en la hoja y el tallo y la pared celular en la hoja en relación a las altas frecuencias. A altos intervalos de poda, se incrementó la fibra neutro detergente en el tallo y todos los constituyentes de la pared celular. Las cenizas totales en la hoja fueron más altas al incrementarse el intervalo de corte, mientras que en el tallo los valores fueron decreciendo.

La composición química entre hojas y tallos fue diferente y se marcaron diferencias muy amplias en todos los constituyentes químicos, con excepción de la concentración de materia seca en la forraje verde. La proteína cruda de la hoja es tres veces superior al contenido en el tallo; la pared celular y la fibra ácida en la hoja es la mitad de lo observado en el tallo. En términos relativos al contenido de fibra ácida, la celulosa foliar es alrededor de un $75 \%$ y en el tallo es de un $80 \%$. La lignina es alrededor de una cuarta parte en la hoja y una quinta parte en el tallo. La morera contiene en sus hojas el doble y hasta el triple de cenizas totales que en el tallo. Piccioni (1970) informa que la materia seca de las hojas de morera contienen $16 \%$ de proteína cruda, $4,1 \%$ de extracto etéreo, $6,9 \%$ de celulosa y $11 \%$ de cenizas. Rodríguez et al. (1994) trabajando con frecuencias de poda y niveles de fertilización nitrogenada encontraron concentraciones de proteína cruda en las hojas de $21 \%$ sin aplicación de fertilizante y $24 \%$ con la aplicación de $80 \mathrm{~kg} / \mathrm{ha}$ de nitrógeno después de cada poda realizada a seis semanas de crecimiento. Al incrementar la frecuencia a 12 semanas, el porcentaje de proteína bajó a $18 \%$ sin fertilización y a $16 \%$ en el nivel de $80 \mathrm{~kg}$ de N. Eswara y Raddy (1992) informaron valores de materia seca y proteína cruda en la hoja de $30 \%$ y $14,5 \%$ respectivamente. 


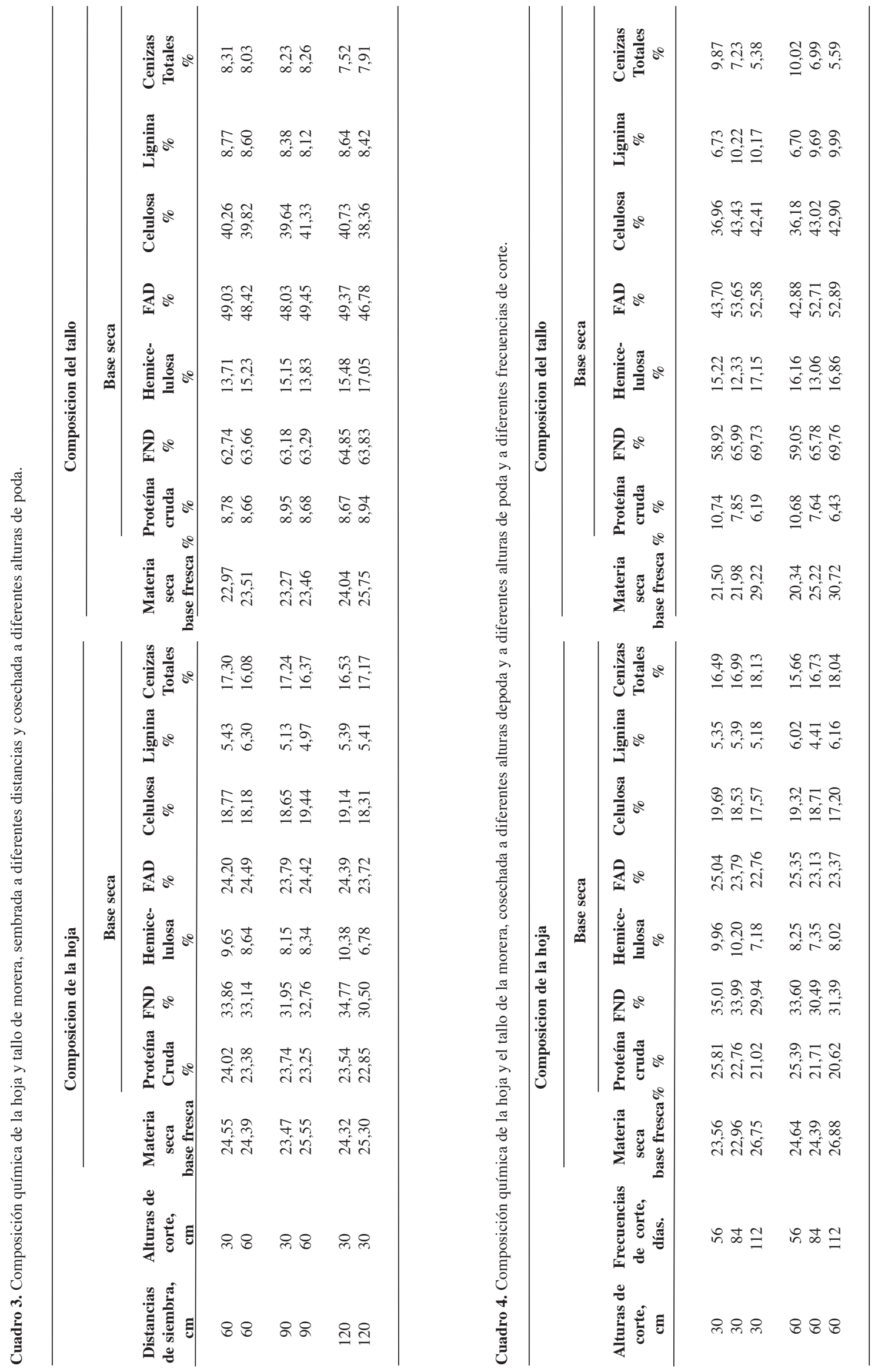




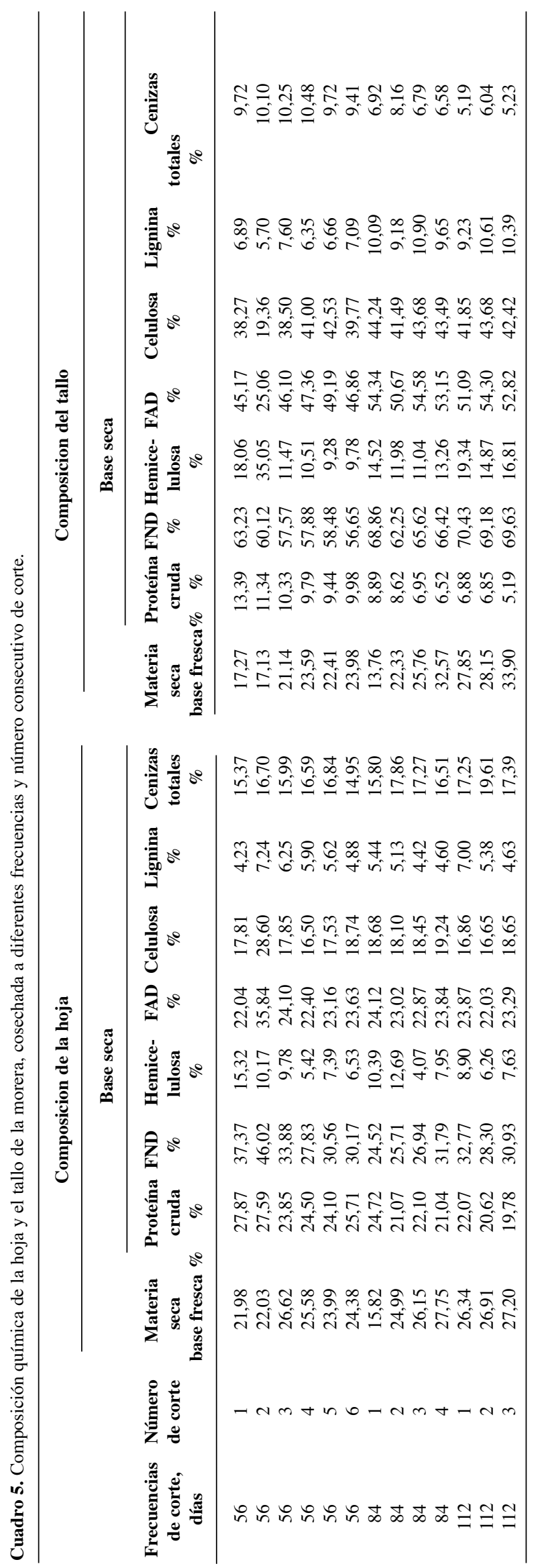

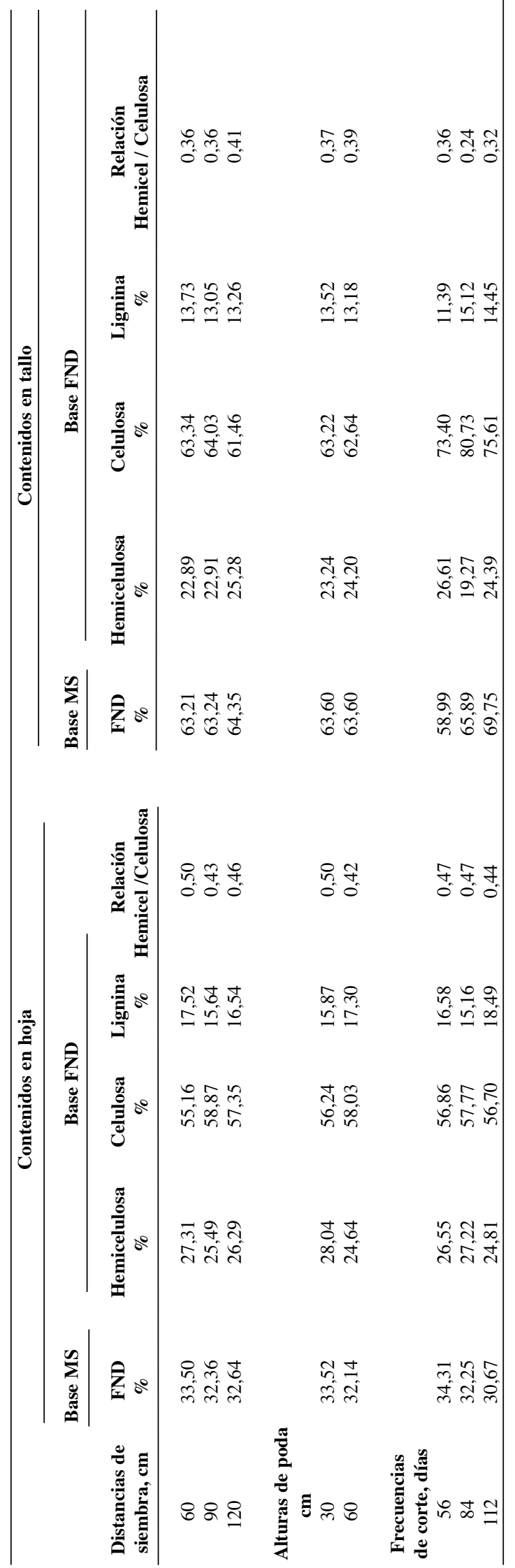


Al comparar la morera con los pastos subtropicales y tropicales, encontramos que es superior en proteína cruda a la alfalfa (17\%) y al orchard grass joven (15\%) o al pasto pangola (11\%) y al elegante (9\%). La alfalfa y el orchard tienen 40 y $55 \%$ de fibra neutro detergente respectivamente, y el pangola y el elefante 70$72 \%$ (Van Soest, 1992), mientras que la hoja de morera tiene $33 \%$ y el tallo $64 \%$. Este último a 112 días de crecimiento tiene una cantidad de pared celular menor o igual a cualquier pasto tropical. La lignina presente en los forrajes subtropicales oscila entre 4 y $8 \%$ y en los tropicales entre 7 y $8 \%$. La morera posee un valor medio de $5,5 \%$ en hoja y $8 \%$ en tallo. Se puede inferir que la hoja de morera sigue siendo superior a cualquier forraje subtropical y el tallo esta en el nivel normal de los pastos tropicales. Si estimamos la energía neta para lactancia en ganado vacuno (Weiss, 1998 y Traxler, M.J. et al., 1998) basados en el calculo de TND y este a partir de la concentración de lignina en la fibra neutro detergente o en la fibra ácido detergente, se confirma la alta digestibilidad de la morera (Rojas y Benavides, 1994; Esguara y Reddy, 1992). En el Cuadro 6 se presenta el cálculo de la concentración de hemice-lulosa, celulosa y lignina en la fibra neutro detergente de hojas y tallos, separadamente. La lignina representa entre el 15 y el $18 \%$ de la pared celular en la hoja y entre el 11 y $15 \%$ de la pared celular en el tallo, lo cual sirve para estimar una alta digestibilidad en la morera, tanto en hojas como en tallos. La limitación que presenta el tallo como alimento animal, no esta relacionada con la calidad química propiamente, sino con la forma física de suministrarla a los animales para su aprovechamiento. Muchos alimentos concentrados que se emplean en la alimentación de animales de carne en el trópico, durante las épocas secas, tienen igual o inferior calidad que el tallo de morera. La hoja debidamente secada y molida, es de una calidad excelente y tiene las propiedades bromatológicas deseadas para ser incluida como ingrediente en las mezclas balanceadas para vacas de alta producción lechera.

\section{CONCLUSIONES Y RECOMENDACIONES}

Los contenidos de proteína cruda y pared celular de la hoja de morera, así como la proporción de los carbohidratos estructurales y la acumulación de cenizas, indican que es un excelente alimento para el ganado de alta producción, pudiendo ser suministrado tanto en fresco como incorporado en forma seca dentro de una dieta balanceada. La composición de los tallos es muy similar a las gramíneas establecidas en las explotaciones tropicales. Los factores experimentados influyeron sobre las proporciones químicas de la materia seca. La distancia de siembra y la altura de poda produjeron pequeñas variaciones en la composición de la hojas y en los tallos de la morera. La frecuencia de corte produjo cambios mayores de los contenidos estudiados; sin embargo, las diferencias en materia seca, proteína, carbohidratos estructurales o en las cenizas totales de las hojas no fueron superiores al 3\% entre la menor y mayor frecuencia. En el tallo los cambios mayores fueron de un $9 \%$ y se produjeron en los contenidos de materia seca, pared celular y en algunos de sus constituyentes estructurales como la celulosa. El número de corte durante el ciclo anual de producción mostró cambios en los componentes químicos, los cuales fueron de magnitud importante para ser considerados apropiadamente, cuando se ofrece la morera a los animales para su consumo. Bajo condiciones tropicales, se debe estudiar la producción de morera a escala industrial para incorporarla como materia prima en la elaboración de alimentos concentrados.

\section{LITERATURA CITADA}

A.O.A.C. 1980. Methods of analysis. Ed. 13. Washington D.C. EUA. Association of official analysis chemistry.

BENAVIDES, J.; LACHAUX, M.; FUENTES, M. 1994. Efecto de la aplicación de estiércol de cabra en el suelo sobre la calidad y producción de biomasa de Morera (Morus sp.). Arboles y arbustos forrajeros en América Central. Serie Técnica. CATIE. Informe Técnico No 236. Volumen II. p. 495-514.

BOSCHINI, C.; DORMOND, H.; CASTRO, A. 1998. Producción de biomasa de morera (Morus alba) en la Meseta Central de Costa Rica, establecida y cosechada a diferentes distancias de siembra, alturas y frecuencias de poda. Agronomía Mesoamericana 9(2):28-39.

CASTRO, A. 1989. Producción de leche de cabras alimentadas con King grass (Pennisetum purpureum $\mathrm{x}$ P. typloides), suplementales con diferentes niveles de follaje de Poró (E. poeppigrama) y de fruto de plátano va de $(\mathrm{Mu}$ sa sp. var. Pelipita). Tesis M.Sc. Turrialba, C.R. UCR/CATIE. 58 p.

CASTRO, A.; BENAVIDES, J. 1994. Evolución de los sistemas de alimentación en explotaciones caprinas de Costa Rica. Arboles y Arbustos Forrajeros en América Central. Serie Técnica. CATIE. Informe Técnico No 236. Volumen II. p. 245-250.

ESWARA, P.; REDDY M.R. 1992. Nutritive value of Muberry (Morus alba) leaves in goats and sheep. Indian Journal of Animal Science 8(4):295-296.

GOERING, H.K.; VAN SOEST, P.J. 1970. Forrage fiber analysis (Apparatus, Reagents, procedures and some aAplications). Agricultural Handbook No. 379. ARSUSDA, Washington, DC. 
ORTIZ, G. 1992. Efecto de la alimentación con pasto King grass. (Pennisetum purpureum $\mathrm{x}$ P. typohoides), suplementado con diferentes niveles de follaje de Morera (Morus alba) y de banano verde (Musa sp) sobre la producción de leche de cabra. Tesis Licenciatura. Escuela de Zootecnia. Universidad de Costa Rica. 45 p.

PICCIONI, M. 1970, Diccionario de Alimentación Animal. Editorial Acribia. Zaragoza. pp. 492-494.

RODRÍGUEZ, C.; ARIAS, R. ; QUIÑONES J. 1994. Efecto de la frecuencia de poda y el nivel de fertilización nitrogenada, sobre el rendimiento y calidad de la biomasa de Morera (Morus sp) en el trópico seco de Guatemala. Arboles y arbustos Forrajeros en América Central. Serie Técnica. CATIE. Informe Técnico No 236. Volumen II. pp. 515-528.

ROJAS, H.; BENAVIDES, J. 1994. Producción de leche de cabras alimentadas con pasto y suplementadas con altos niveles de morera (Morus sp). Arboles y arbustos forrajeros en América Central. Serie Técnica. CATIE. Informe Técnico No. 236. Volumen I. pp. 305-319.
SAS. 1985. Statistical Analysis System. SAS User's Guide: Statistics (Version 5 Ed.) SAS Institute Inc. Cary, NC.

TRAXLER, M.J.; FOX, D.G.; VAN SOEST, P.J.; PELL, A.N.; LASCANO, C.E.; LANNA, D.P.D.; MOOR, J.E.; LANA, R.P, VELEZ, M.; FLORES, A. 1998. Predicting forraje indigestible NFD from lignina concentration. Journal of Animal Science 76(5):1469-1480.

VAN SOEST, P.J. 1982. Nutritional ecology of the ruminant. Durham and Downey, Inc. Portland. USA.

VASQUEZ, A. 1982. Estudio detallado de los suelos de la Estación Experimental de Ganado Lechero El Alto. Escuela de Fitotecnia, Facultad de Agronomía, Universidad de Costa Rica. p. 36.

WEISS, W.P. 1998. Estimating the available energy content of feeds for dairy cattle. Journal of Dairy Science 81(3):830-839. 